\title{
Feature Extraction of Gas Sensor Response Based on Subspace-Based Identification
}

\author{
Yoshinori Takei ${ }^{1,2, *}$, Hidehito Nanto ${ }^{1,2}$ and Kiyoshi Wada ${ }^{2}$ \\ ${ }^{1}$ Department of Robotics, Kanazawa Institute of Technology, \\ 7-1 Ohgigaoka, Nonoichi, Ishikawa 921-8501, Japan \\ ${ }^{2}$ AMS R\&D Center, Kanazawa Institute of Technology, \\ 3-1 Yatsukaho, Hakusan, Ishikawa 924-0838, Japan
}

(Received October 7, 2013; accepted February 4, 2014)

Key words: gas sensor, subspace identification method, Prony's method

In this paper, feature extraction based on a subspace-based identification technique for a transient response of a semiconductortype gas sensor is proposed. A typical gas sensor response can be interpreted as the sum of step responses of the first- or highorder lag system, and we have investigated the feature extraction method of the sensor output, which can be approximated by Prony's method. The method gives the time constant and gain parameters of the sensor response, which includes useful information for a discrimination of sample gases. In this paper, we show a feature extraction method based on a subspace-based identification for the step response of the sensor. The sum of exponentials model in Prony's method can be represented as a state space model. Then, the system matrices can be estimated by the multi-input multi-output (MIMO) output error state space model identification (MOESP)-like procedure. Numerical simulation shows that the proposed algorithm can be effective in feature extraction, and the method can be applied to the sensor response to mixture gases.

\section{Introduction}

Odor sensing systems combining gas sensors with multivariate analysis (MVA) methods, called electric nose systems, ${ }^{(1)}$ have been paid much attention in various areas. Electric nose systems generally have many gas sensors as elements in the sensor array to discriminate odorants, and semiconductor-type gas sensors are suitable sensors as the element because of their stability and other advantages. The gas sensor in electric nose systems is basically expected to have a high selectivity and sensitivity. Semiconductor gas sensors, however, have a low selectivity; therefore, a feature extraction method from the transient response of the sensor ${ }^{(2)}$ has been proposed to improve the selectivity in the MVA by the augmentation of the feature vector.

${ }^{*}$ Corresponding author: e-mail: takei@neptune.kanazawa-it.ac.jp 
In this paper, feature extraction based on a subspace-based identification technique for a transient response of a semiconductor-type gas sensor is considered. A typical gas sensor response can be interpreted as the sum of step responses of the first- or high-order lag system; thus, we have investigated the feature extraction method of the sensor output, which can be approximated by Prony's method. ${ }^{(3)}$ The method gives the time constant and gain parameters of the sensor response, which includes useful information for a discrimination of sample gases.

This paper shows a feature extraction based on a subspace-based identification method, called the multi-input multi-output (MIMO) output error state space model identification (MOESP) method ${ }^{(4,5)}$ for the step response of the sensor. The sum of exponentials model in Prony's method can be represented as a discrete time state space model. Then, the system matrices can be estimated by the MOESP-like procedure. Numerical simulation shows that the proposed algorithm can be effective in feature extraction, and the method can be applied to the sensor response to mixture gases.

\section{Review of Sensor Response Modeling}

Assuming that the gas reaction on the surface of the sensor can be regarded as a firstorder reaction, the transient response of the sensor can be modeled as the sum of the step response of the discrete first-order lag system. Then, we have

$$
\tilde{y}_{k}=\sum_{i=1}^{n} C_{i}\left(1-r_{i}^{k}\right),
$$

where

$$
r_{i}=\exp \left(-\Delta t / \tau_{i}\right)
$$

$\Delta t$ is the sampling period, $\tau$ is a time constant and $C$ is a steady-state value that means the initial concentration or saturation mass of adsorption. $n$ is the order of the model, which means the number of gas components. Strictly speaking, even if a single gas is provided to the sensor, the order of the sensor response model is not first-order. The dominant parameters that should be extracted, however, are obtained from the model with $n=1$. Therefore, the order of the model can be treated as the number of gas components.

The main problem is to obtain the above parameters of the model, that is, $\tau$ and $C$ in eq. (1). First, we consider the exponential function fitting in Prony's method ${ }^{(3)}$ to approximate the sensor response. To obtain $\tau$ and $C$, the sensor output model (1) can be represented as the sum of exponentials. Then, using the following $L$-step ahead differential,

$$
y_{k}=\tilde{y}_{k+L}-\tilde{y}_{k},
$$

the sum of exponentials can be introduced to rewrite the output 


$$
y_{k}=\sum_{i=1}^{n} m_{i} r_{i}^{k} .
$$

The represented gain $m$ and $C$ are related as

$$
m_{i}=C_{i}\left(1-\exp \left(-L \Delta t / \tau_{i}\right)\right), \quad(i=1, \ldots, n)
$$

then, we consider the estimations of $\tau$ and $C$ through the identification of the model shown in eq. (4).

\section{Subspace-Based Prony's Method}

In this section, we show the estimation method of the sum of exponentials based on the subspace-based Prony's method. Equation (4) added the noise term $e_{k}$, which can be represented as the discrete time state space model:

$$
\begin{gathered}
\boldsymbol{x}_{k+1}=\boldsymbol{A} x_{k}, \\
\boldsymbol{y}_{k}=\boldsymbol{c}^{T} x_{k}+\boldsymbol{e}_{k},
\end{gathered}
$$

where the system matrices $A$ and $c$ and the initial state vector $x_{0}$ are

$$
\begin{gathered}
\boldsymbol{A}=\left[\begin{array}{llll}
r_{1} & & & 0 \\
& r_{2} & & \\
& & \ddots & \\
0 & & & r_{n}
\end{array}\right], \\
\boldsymbol{c}^{T}=\left[\begin{array}{llll}
m_{1} & m_{2} & \cdots & m_{n}
\end{array}\right], \\
\boldsymbol{x}_{0}=\left[\begin{array}{llll}
1 & 1 & \cdots & 1
\end{array}\right] .
\end{gathered}
$$

Here, the stacked vector defined as follows is introduced:

$$
y_{i}(k+N) \triangleq\left[y_{k+N}^{T}, y_{k+N+1}^{T}, \cdots, y_{k+N+i-1}^{T}\right]^{T},
$$

where $y_{i}(k+N) \in \mathbb{R}^{i} . i$ is the auxiliary order and $N$ denotes the data length. The stacked vector of the noise $e_{i}(k+N)$ can be defined similarly. Then, the Hankel matrix by using the stacked vectors is defined as

$$
\boldsymbol{Y}_{k, i, N} \triangleq\left[y_{i}(k) y_{i}(k+1), \cdots, y_{i}(k+N-1)\right]
$$


Corresponding definitions for the noise $\boldsymbol{E}_{k, i, N}$ are also defined. In relation to the order of the system $n$, the pair $i$ and $N$ satisfy $i>n$ and $N \gg n$. Defining the state vector sequence as

$$
\boldsymbol{X}_{\mathrm{k}, \mathrm{N}} \triangleq\left[x_{k}, x_{k+1}, \cdots, x_{k+N-1}\right]
$$

we obtain the following data equation:

$$
\boldsymbol{Y}_{k, i, N}=\boldsymbol{\Gamma}_{i} \boldsymbol{X}_{k, N}+\boldsymbol{E}_{k, i, N}
$$

$\Gamma_{i}$, called the extended observability matrix, is defined as

$$
\boldsymbol{\Gamma}_{i}=\left[\begin{array}{c}
c^{T} \\
c^{T} \boldsymbol{A} \\
\vdots \\
c^{T} \boldsymbol{A}^{i-1}
\end{array}\right]
$$

The key problem of the subspace method is to estimate the column space of $\boldsymbol{\Gamma}_{i}$ including the system matrices.

To extract the column space of $\boldsymbol{\Gamma}_{i}$, compute the $\mathrm{SVD}^{(6)}$ of the matrix $\boldsymbol{Y}_{k, i, N}$ given in eq. (16), i.e.,

$$
\boldsymbol{Y}_{i, k, N}=\left[\boldsymbol{U}_{n} \boldsymbol{U}_{n}^{\perp}\right]\left[\begin{array}{cc}
\Sigma_{n} & O \\
O & \Sigma_{2}
\end{array}\right]\left[\begin{array}{c}
\boldsymbol{V}_{n}^{T} \\
\left(\boldsymbol{V}_{n}^{\perp}\right)^{T}
\end{array}\right],
$$

where " $\perp$ " denotes perpendicular and the matrix $\boldsymbol{\Sigma}_{i}$ is diagonal. The matrix $\boldsymbol{U}_{n} \in \mathbb{R}^{i \times n}$ gives the estimate of $\boldsymbol{\Gamma}_{i}$. The order of the matrix $\Sigma_{n}$ can be equal to that of the system and $\boldsymbol{\Sigma}_{2}$ is zero matrix when $e_{k}=0$. In this case, $\Sigma_{2}$ has the same singular values smaller than the $\mathrm{n}$-th largest singular value on the diagonal. Note that to evaluate the obtained singular values, the order of the system, that is, the number of components of gases should be determined.

Using the matrix $\boldsymbol{U}_{n}$ given in eq. (16), solve the following set of equations to estimate the system matrices $\hat{\boldsymbol{A}}$ and $\hat{\boldsymbol{c}}$.

$$
\begin{gathered}
\hat{\boldsymbol{c}}=\boldsymbol{U}_{n}(1,:) \\
\boldsymbol{U}_{n}^{(1)} \hat{\boldsymbol{A}}=\boldsymbol{U}_{n}^{(2)}
\end{gathered}
$$

In eq. (17), the submatrix of $\boldsymbol{U}_{n}$ is denoted by using a MATLAB-like notation. $\boldsymbol{U}_{n}^{(1)}$ is the submatrix composed of the first $(i-1)$ rows of the matrix $\boldsymbol{U}_{n}$, and $\boldsymbol{U}_{n}^{(2)}$ is constructed using the last rows in a similar way. Equation (18) expresses the shift-invariance property in the estimate $\boldsymbol{U}_{n}$. The time constant $\tau$ can be estimated by applying the eigenvalue decomposition (EVD) to $\hat{\boldsymbol{A}}$. 
Once the time constants can be obtained, then we can estimate the gain $\mathrm{m}$ to minimize the following cost function

$$
J_{N}=\sum_{k=0}^{N}\left(y_{k}-\sum_{k=1}^{n} m_{i} r_{i}^{k}\right)^{2}
$$

Then, we have

$$
\boldsymbol{m}_{n}=\left(\boldsymbol{R}_{N}^{T} \boldsymbol{R}_{N}\right)^{-1} \boldsymbol{R}_{N} \boldsymbol{y}_{N}
$$

where

$$
\begin{gathered}
\boldsymbol{m}_{n}=\left[\begin{array}{llll}
m_{1} & m_{2} & \cdots & m_{n}
\end{array}\right]^{T}, \\
\boldsymbol{y}_{N}=\left[\begin{array}{llll}
y_{0} & y_{1} & \cdots & y_{N-1}
\end{array}\right]^{T}, \\
\boldsymbol{R}_{N}=\left[\begin{array}{cccc}
1 & 1 & \cdots & 1 \\
r_{1} & r_{2} & \cdots & r_{n} \\
\vdots & \vdots & \ddots & \vdots \\
r_{1}^{N-1} & r_{2}^{N-1} & \cdots & r_{n}^{N-1}
\end{array}\right] .
\end{gathered}
$$

\section{Numerical Example}

We consider the single-input single-output (SISO) system with the following parameters, which means that the single gas sensor responded to the mixture gas.

$$
\begin{gathered}
\tau=\{20,5,50\} \\
C=\{3,5,2\}
\end{gathered}
$$

The output data length $N=5000$ and the sampling period $\Delta t=0.05$. The differential interval and the auxiliary order are chosen as $L=900$ and $i=1500$, respectively. Note that the auxiliary order is chosen as a large number in comparison with the system order. The noise sequence $e_{k}$ is a realization of white Gaussian noise with zero mean, and the variance of the noise is adjusted as the output $\mathrm{SNR}=35 \mathrm{~dB}$. Figure 1 shows the simulated sensor response (solid line) with the noise and the response of the component outputs (dashed line). The problem is to estimate the parameter pairs of $(\tau, C)$ from the sensor output data denoted by the solid line in Fig. 1. Applying the proposed method, one example of the obtained estimates of $(\tau, C)$ is as follows. 


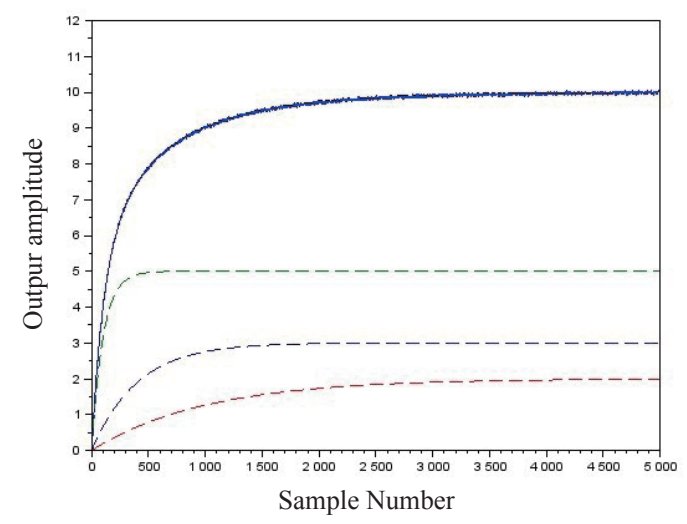

Fig. 1. (Color online) Simulated sensor output (solid) and component outputs (dashed).

$$
\begin{aligned}
& \tau=\{50.85,20.63,5.020\} \\
& C=\{1.907,3.049,5.036\}
\end{aligned}
$$

The parameters are sorted by $\tau$. It shows that the feature parameter of each component can be estimated satisfactorily. Then, the common logarithm of the singular value matrix $\Sigma_{i}$ shown in eq. (16) can be shown in Fig. 2. Figure 2 shows the first ten largest singular values only, and we can see that the matrix has three large singular values, that is, the number of gas components can be determined as three. Figures 3 and 4 show the histogram plots of the estimates $(\tau, C)$ obtained under the same condition in 100 trials with different noise realizations. We can see that the time constant and gain parameters can be well estimated. The results show that the proposed algorithm can extract and isolate the feature parameters.

We can readjust user-defined parameters $L$ and $i$ to improve the estimates $(\tau, C)$ under a low SNR condition; however, the estimates can be inaccurate and we have to consider a more rational guideline to determine the parameters $L$ and $i$ in future work.

\section{Conclusions}

In this paper, we have considered the feature extraction method based on a subspacebased identification for the step response of the sensor. The sum of exponentials model in Prony's method could be represented as a discrete time state space model. We have derived the subspace-based Prony's method by the MOESP-like procedure. Numerical simulations have shown that the proposed algorithm can be effective in feature extraction, and the possibility of feature extraction for the sensor response to mixture gases. 


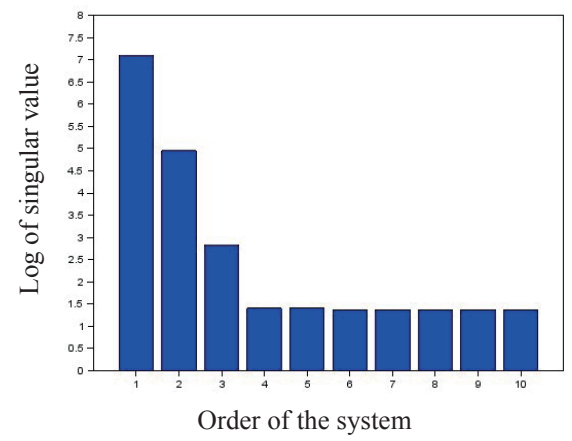

Fig. 2. (Color online) Results of singular value decomposition.
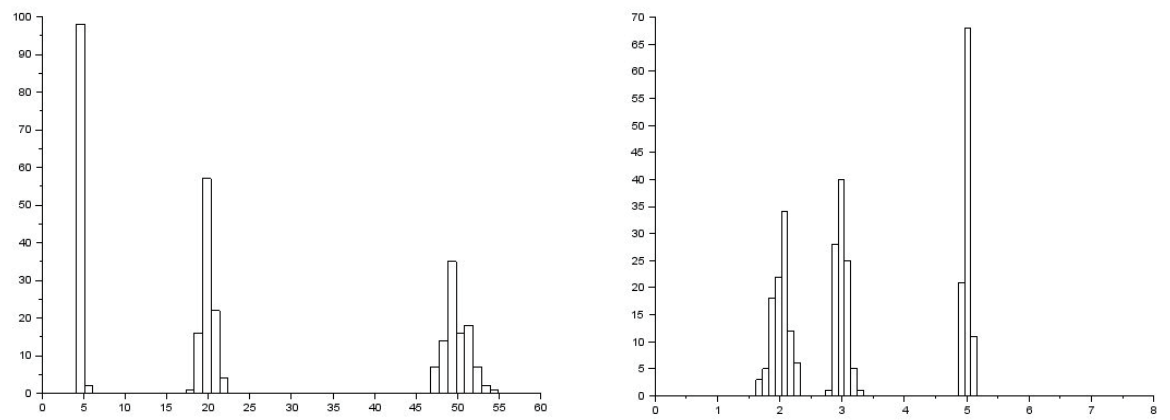

Fig. 3 (left). Histogram plots of estimated time constants ( $\tau=5,20$, and 50$)$.

Fig. 4 (right). Histogram plots of estimated steady-state values $(C=2,3$, and 5$)$.

\section{Acknowledgements}

This work was partly supported by JSPS KAKENHI Grant Number 23760394.

\section{References}

1 T. C. Pearce, S. S. Schiffman, H. T. Nagle and J. W. Gardner: Handbook of Machine Olfaction (Wiley-VCH, Weinheim, 2003).

2 S. Seto, H. Kawabe, L. Shi, Y. Shimomura, T. Oyabu and T. Katsube: Sens. Mater. 18 (2006) 1.

3 Y. Takei, S. Koyama, H. Nanto and T. Mihara: Sens. Mater. 21 (2009) 4.

4 M. Verhaegen and P. Dewilde: Int. J. Control 56 (1992) 1187.

5 M. Verhaegen: Int. J. Control 58 (1993) 555.

6 G. H. Golub and C. F. Van Loan: Matrix Computations (Johns Hopkins University Press, Baltimore and London, 1991) 3rd ed. 